\title{
A Single Nucleotide Polymorphism in Inflammatory Gene RNASEL Predicts Outcome After Radiation Therapy for Localized Prostate Cancer
}

\author{
Jonathan D. Schoenfeld ${ }^{1}$, Danielle N. Margalit ${ }^{2,3}$, Julie L. Kasperzyk ${ }^{2,4}$, Irene M. Shui ${ }^{4}$, \\ Jennifer R. Rider ${ }^{2,4}$, Mara M. Epstein ${ }^{4}$, Allison Meisner ${ }^{4}$, Stacey A. Kenfield ${ }^{4}$, Neil E. \\ Martin $^{2,3}$, Paul L. Nguyen ${ }^{2,3}$, Philip W. Kantoff ${ }^{2,3}$, Edward L. Giovannucci ${ }^{4}$, Meir J. \\ Stampfer ${ }^{2,3,4}$, and Lorelei A. Mucci ${ }^{4}$ \\ ${ }^{1}$ Harvard Radiation Oncology Program, Boston, MA \\ ${ }^{2}$ Brigham and Women's Hospital, Boston, MA \\ ${ }^{3}$ Dana-Farber Cancer Institute, Boston, MA \\ ${ }^{4}$ Harvard School of Public Health, Boston, MA
}

\begin{abstract}
Purpose-To study associations between single nucleotide polymorphisms (SNPs) in RNASEL, a gene implicated in inflammation and prostate cancer risk, and outcomes following radiation therapy (RT).

Experimental Design-We followed participants in the prospective US Health Professionals Follow-Up Study treated with RT for early-stage prostate cancer. Three SNPs were genotyped based on previously determined functional and biological significance. We used multivariable Cox proportional hazards models to assess per-allele associations with the primary outcome defined as time to a composite endpoint including development of lethal prostate cancer or biochemical recurrence.
\end{abstract}

Results-We followed 434 patients treated with RT for a median of 9 years. On multivariate analysis, the rs 12757998 variant allele was associated with significantly decreased risk of the composite endpoint (HR: $0.65 ; 95 \%$ CI: $0.45-0.94 ; \mathrm{p}=0.02$ ) driven by decreased biochemical recurrence (HR: $0.60 ; 95 \%$ CI: $0.40-0.89 ; \mathrm{p}=0.01$ ) and men treated with external beam (HR: $0.58 ; 95 \%$ CI: $0.36-0.93 ; p=0.02$ ). In contrast, in 516 men from the same cohort treated with radical prostatectomy, we found no significant impact of this variant on outcome. Furthermore, the rs 12757998 variant allele significantly modified the association between androgen deprivation therapy and outcomes following RT (p-interaction $=0.02)$.

Conclusion-We demonstrate an association between RNASEL SNP rs12757998 and outcome after RT for prostate cancer. This SNP is associated with increased circulating C-reactive protein and interleukin-6, suggesting a potential role for inflammation in the response to radiation. If validated, genetic predictors of outcome may help inform prostate cancer management.

CORRESPONDING AUTHOR: Jonathan D. Schoenfeld, MD, MPhil, MPH, Department of Radiation Oncology, Brigham and Women's Hospital, 75 Francis Street, ASB1-L2, Boston, MA 02115, Phone: 617-732-6231, Fax: 617-975-0932,

jdschoenfeld@partners.org.

DISCLAIMERS: There are no conflicts of interest to disclose

Presented at the American Society for Radiation Oncology 54th Annual Meeting, October 28 - 31, 2012, Boston, MA. 


\section{Keywords}

prostate cancer; radiotherapy; inflammation; outcome; single nucleotide polymorphism

\section{Introduction}

Radiation therapy in prostate cancer, either external beam or brachytherapy, is an effective therapy for men with early-stage, organ confined prostate cancer $(1,2)$. However, not all patients are cured, some because of occult metastases at the time of treatment and others because of failure of local control of cancer. In either scenario, cure or failure to cure may be driven by both host and somatic factors. More specifically, the role of inherited genetic variation to predict outcome after radiation therapy is biologically plausible and has been investigated somewhat in radiation therapy (3-5). Most studies have focused on polymorphisms in DNA damage response genes and examined toxicity outcomes (6-9). In contrast, data regarding the effects of genetic variation on treatment-specific cancer outcomes are more limited.

Ribonuclease $\mathrm{L}$ ( $R N A S E L$ ) is compelling as a potential modulator of response to radiation therapy in prostate cancer given its role in inflammation and response to infection. $R N A S E L$ is a putative tumor-suppressing enzyme that may inhibit growth and proliferation and induce apoptosis (10-12), and is implicated in the cleavage of viral RNA and the interferonmediated immune response (13). Polymorphisms in RNASEL, located on chromosome 1q25, have been inconsistently linked to hereditary and general prostate cancer risk (14-26). In particular, the minor variant of single nucleotide polymorphism (SNP) rs12757998, located immediately downstream of the $R N A S E L$ coding region, was recently associated with an increased risk of prostate cancer and specifically higher-grade tumors (19), and was associated with elevated serum levels of the inflammatory markers C-reactive protein (CRP) and interleukin (IL)-6 (19).

The role of inflammation and the immune system in radiation-induced cancer cell death is complex and incompletely defined $(27,28)$. Preclinical data suggest that, at least under certain circumstances, the immune system may augment cytotoxic effects of radiation therapy and may even be required for tumor eradication $(29,30)$. Conformal radiation therapy given to prostate cancer patients induces a local inflammatory response and has been demonstrated to increase circulating IL-6 levels (31-34). Therefore, we hypothesized that by modulating this response, inherited variation in $R N A S E L$ could affect prostate cancer outcomes following definitive radiation therapy for early-stage prostate cancer and sought to explore this association among men with prostate cancer in the US Health Professionals Follow-Up Study.

\section{Methods}

\section{Study population}

The prospective Health Professionals Follow-Up Study (HPFS) enrolled 51,529 male medical professionals in 1986 to investigate the causes of cancer and heart disease. These cohort participants are subsequently followed with biennial questionnaires designed to collect information about medical diagnoses and lifestyle factors. Response rates to these surveys are high (approximately 96\%). Between 1993 and 1995, 18,018 participants provided a blood sample for analysis. The study base for this project was men in the blood cohort who were diagnosed with prostate cancer between $1993-2010$. 
The institutional review board at the Harvard School of Public Health approved the HPFS study. Participants provided written informed consent for review of their medical records.

\section{Genotype Assessment}

We characterized genetic variation for three SNPs within the RNASEL gene: rs486907, rs627928 and rs12757998. These SNPs were selected based on previously established functional significance $(16,19,35-37)$. Genotyping was performed in a blinded fashion after extraction of DNA from whole blood using a standard QIAmp kit (QIAGEN Inc. Chatsworth, CA) protocol using Biotrove Open Array SNP Genotyping Platform at the Harvard Medical School - Partners Healthcare Center for Genetics and Genomics. All SNPs had greater than $90 \%$ completion and the concordance was greater than $99 \%$ for blinded quality control samples. Pair-wise $\mathrm{r} 2$ values for these SNPs in the study population were: rs486907-rs627928 0.61; rs486907-rs12757998 0.27; and rs627928-rs12757998 0.15.

\section{Assessments of prostate cancer outcomes}

Participants initially report a new prostate cancer diagnosis on biennial questionnaires, and cases are confirmed through detailed review of medical records and pathology reports whenever possible $(>90 \%)$. In addition, detailed information on clinical features at diagnosis and initial treatments are abstracted from the review. In HPFS, men with prostate cancer are specifically followed with biennial questionnaires to assess factors related to follow-up treatments, PSA outcomes, clinical progression and development of metastases.

Our primary outcome was a composite progression endpoint consisting of the development of lethal prostate cancer or biochemical recurrence. Lethal prostate cancer was defined as participants who developed bone metastases or experienced a prostate cancer-specific death. We ascertained prostate-specific causes of death using reports from families, as well as postal service and National Death index records. Study physicians then confirmed these deaths by reviewing the available medical records in more than $98 \%$ of cases. Similarly, we determined biochemical recurrence from available medical records and from physician questionnaires based on accepted treatment-specific definitions as described previously (for radical prostatectomy, PSA higher than $0.2 \mathrm{ng} / \mathrm{mL}$ after surgery and for at least 2 consecutive measures; for radiation, an increase of 2 or more $\mathrm{ng} / \mathrm{mL}$ higher than the nadir PSA; for brachytherapy, an increase of 1 or more $\mathrm{ng} / \mathrm{mL}$ higher than the nadir PSA for at least 2 consecutive measures) (38). If records were not available, we also identified recurrence through patient self-reports of PSA increase from participant questionnaires; these accounted for $44 \%$ of biochemical recurrence events. Date of composite failure was defined as the date of the earliest event. Men for whom we could not ascertain the date of biochemical recurrence but who also developed lethal prostate cancer were considered to have failed at the date at which they developed lethal cancer and all men were included in time to biochemical failure analyses, including those who eventually developed lethal prostate cancer.

\section{Population for analysis}

We included participants who previously provided a blood sample for genotyping and were then diagnosed with early-stage (T1-3N0/xM0/x) prostate cancer after enrollment in HPFS. To reduce the possibility of population stratification we limited our study to Caucasians, who account for the majority (95\%) of HPFS participants. We limited our study to men with disease most likely to be curable with local therapy at the time of diagnosis by further excluding participants with an initial PSA greater than $50 \mathrm{ng} / \mathrm{mL}$ or men in whom lethal prostate cancer was identified less than one year following their diagnosis. Our primary analyses involved men initially treated with any form of definitive radiation therapy, but who did not receive a radical prostatectomy. To determine whether the association could be 
generalized to another outcome group, we then evaluated men who were initially treated with radical prostatectomy and not radiation therapy, but who otherwise satisfied all of the same inclusion and exclusion criteria.

\section{Statistical Analyses}

We examined variation in $R N A S E L$ and participant characteristics at diagnosis using Pearson correlations (age at diagnosis) and Chi-Squared trend tests (PSA, Gleason score, clinical stage). We undertook time to event analyses, calculating follow-up time from date of treatment initiation to date of the composite outcome and the individual components of lethal prostate cancer and biochemical recurrence. Men were censored if they died of other causes or at the end of the study (December 2011). We used Cox proportional hazard regression models to calculate maximum likelihood hazard ratios and $95 \%$ confidence intervals of the association between variation and RNASEL and cancer outcomes. We analyzed genetic effects using the additive model, considering the presence of 0,1 or 2 copies of the minor allele as an ordinal variable. In multivariable models, we adjusted for PSA at diagnosis (continuous; ng/mL), clinical stage (T1/T2 versus T3), Gleason score ( $<7$ versus 7 versus >7) as well as age at treatment to examine independent associations of SNPs on cancer outcomes. We also conducted exploratory analyses including multiplicative interaction terms in our adjusted models to evaluate whether the associations of $R N A S E L$ SNPs differed by participant characteristics at diagnosis (age, Gleason score, PSA, stage) and whether variation in SNP rs12757998 modified the associations between neoadjuvant and/or adjuvant androgen deprivation therapy use and outcome.

All reported p-values are 2 -sided, with $\mathrm{p}<0.05$ considered statistically significant. SAS version 9.1 (SAS institute Inc, Cary, North Carolina) was used for all analyses.

\section{Results}

\section{Participant and Treatment Characteristics}

We included 434 men who received radiation therapy and were diagnosed with prostate cancer and treated between 1992 and 2011 in our main analysis (Supplementary Figure 1). Patient characteristics are shown in Table 1. The median age at diagnosis and treatment was 72 years. Most participants were diagnosed with early-stage (T1/T2) disease (98\%) with Gleason scores less than 7 (69\%) and PSA levels less than $10 \mathrm{ng} / \mathrm{mL}$ (73\%). One-hundred seventy three patients (40\%) received androgen deprivation as a component of their initial treatment and 191 patients (44\%) received brachytherapy, forty-eight of who also received external beam radiation.

The distribution of allelic variation in the three genotyped RNASEL SNPs is shown in Table 1. There were no significant associations between the presence of allelic variants and age at treatment, PSA at diagnosis, Gleason score or clinical stage (data not shown).

\section{Outcomes}

With a median follow-up of 9 years through 2011, 100 (23\%) men had a recurrence or developed lethal disease (Table 1). Among these 100 men, 9 developed lethal prostate cancer without recorded biochemical recurrence, 77 had a biochemical recurrence only, and 14 had a biochemical recurrence and developed lethal prostate cancer. Among men who experienced outcomes, median time to the composite endpoint, lethal prostate cancer and biochemical recurrence was $4.3,7.3$ and 4.3 years, respectively. Time to biochemical recurrence was similar in the 14 men who eventually developed lethal cancer and the 77 men who did not (4.1 years compared with 4.3 years). Time to lethal prostate cancer was 7.8 years in the 14 men with previously detected biochemical recurrence as compared with 6.4 
years in the 9 men without detected biochemical recurrence. For the 334 men who remain progression free, the follow-up was 9.8 years. Among these men, 80 were censored due to deaths unrelated to prostate cancer.

Univariate associations are shown in Supplementary Table 1. SNP rs12757998, but not rs 486907 or rs627928, was associated with a significantly prolonged time to the composite outcome (HR 0.70; 95\% CI 0.50 - 0.99; p = 0.04). Examination of median time-to-event stratified by genotype for men who reached the composite outcome did not suggest that this result was driven by a small number of outliers with an exceptionally long time to failure (Supplementary Figure 2). This association of rs12757998 remained significant after adjusting for patient age at treatment, Gleason score, PSA at diagnosis and clinical stage (Table 2; HR 0.65; 95\% CI $0.45-0.94 ; \mathrm{p}=0.02$ ). None of the SNPs had a significant effect on time to the development of lethal prostate cancer. Only rs 12757998 had a significant effect on time to biochemical recurrence (HR 0.60; 95\% CI $0.40-0.89 ; p=0.01$ ). In contrast, as a control to assess the specificity of the effect to radiation therapy, we examined the effect of SNP rs 12757998 in 516 participants from the Health Professionals cohort with early-stage prostate cancer who were treated definitively with radical prostatectomy and did not receive radiation therapy. Among these 516 patients treated with radical prostatectomy, $94(18 \%)$ reached the composite outcome. In these men, rs 12757998 was not associated with time to the composite outcome (multivariate HR $0.87 ; 95 \%$ CI $0.61-1.25 ; \mathrm{p}=0.45 ; \mathrm{p}$ interaction $=0.26$ ) or biochemical recurrence (multivariate HR 0.86; 95\% CI $0.60-1.25 ; \mathrm{p}$ $=0.43 ;$ p-interaction $=0.19$ ).

\section{Interaction and subgroup analyses}

Further exploratory analyses demonstrated no evidence of interaction between allelic variation in rs12757998 and age, PSA at diagnosis, Gleason score and clinical stage on time to the composite outcome ( $p>0.05$, data not shown). However, variation in rs $12757998 \mathrm{did}$ modify the effect of androgen deprivation therapy (Table 3 , p-interaction $=0.02$ ). In men homozygous for the major allele variant, the use of androgen deprivation therapy was associated with a significantly reduced hazard for the composite endpoint and biochemical recurrence (HR 0.38, 95\% CI $0.20-0.72, p=0.003$ and HR 0.42, 95\% CI $0.22-0.79, p=$ 0.007 , respectively). In contrast, associations between androgen deprivation and outcomes were not statistically significant in men with at least one variant rs 12757998 allele. Including this interaction in our multivariate analyses further strengthened associations between variation in rs 12757998 and outcomes $(\mathrm{p}=0.002$ for the composite endpoint and $\mathrm{p}$ $=0.0007$ for biochemical recurrence).

We also evaluated the impact of brachytherapy on the effect of allelic variation in rs 12757998 (Table 2). The effect of allelic variation in rs 12757998 on time to the composite outcome and biochemical recurrence was less pronounced in men who received brachytherapy (HR $0.85,95 \%$ CI $0.46-1.59, \mathrm{p}=0.61$ ) as compared with men treated with external beam radiation only (HR $0.58,95 \%$ CI $0.36-0.93, \mathrm{p}=0.02$ ).

Finally, as rs12757998 has previously been associated with the development of high-grade disease, we evaluated the effects of variation in rs 12757998 on the subset of men with Gleason $\geq 7$ (Table 2). In these participants, the effect of allelic variation on the composite endpoint was particularly apparent in men treated with radiation therapy (HR $0.55,95 \%$ CI $0.31-0.96, \mathrm{p}=0.03$ ) as compared with men who received a radical prostatectomy (HR 1.11, $95 \%$ CI $0.88-1.82, \mathrm{p}=0.69$ ). 


\section{Discussion}

Our prospective study among men with early-stage prostate cancer evaluated whether variation in three SNPs in $R N A S E L$, a gene with previously established biological and functional significance influenced prostate cancer outcomes after radiation therapy. Presence of the minor allele in SNP rs 12757998 conferred a significant reduction in risk of the composite outcome that was driven by a decreased risk of time to biochemical recurrence. This association remained significant after adjusting for known predictors of recurrence including Gleason score, tumor stage and PSA. We found no associations between polymorphisms in the two other SNPs and biochemical recurrence.

We found that the presence of the minor allele variant in rs12757998 was associated with a decreased risk of progression in our analysis. We previously found this variant was associated with an increased risk of prostate cancer overall and specifically of high-grade disease (19). These two effects may illustrate the complex role inflammation and immunity may have in prostate cancer carcinogenesis and its subsequent response to radiation treatment. Although an increased propensity for inflammation may contribute to tumor development, a treatment-induced inflammatory response could also mediate tumor cell death. Indeed, decreased $R N A S E L$ protein levels were recently associated with resistance of prostate cancer cell lines to apoptosis induced by topoisomerase inhibitors and the tumor necrosis pathway (39) and interferon-related genes have been implicated in radiation responsiveness in both cell lines and human patients $(40,41)$. More specifically, we previously showed that rs 12757998 was associated with elevated levels of circulating IL-6 (19), thus potentially influencing the typical cytokine milieu produced by prostate cancer radiation therapy (31-34). This may also explain why variation in rs486907 and rs627928 were not associated with outcome, as these SNPs have limited correlation with rs12757998 and were previously found not to be associated with changes in circulating cytokine levels (19).

In support of the hypothesis that a pro-inflammatory phenotype may contribute to both tumor development and more favorable response to radiation treatment, we observed that the association of variation in RNASEL and the composite outcome of lethal and recurrent prostate cancer was specific to men treated with radiation therapy. Among men in HPFS treated with radical prostatectomy, there was no benefit from the minor variant of rs 12757998 , as might be expected if this effect were mediated by a radiation-induced inflammatory response. Similarly, the effect was reduced in participants treated with brachytherapy, a localized and dose-escalated form of radiation therapy that may have differential effects on the processes that lead to prostate cancer cell death, possibly by delivering higher biologically effective doses that overcome the RNASEL-dependent effects.

The use of inherited genetic information to predict response and guide treatment has been steadily increasing in oncologic practice, and pharmacogenomics, in particular, has been an active area of research (42). However, very limited data are available suggesting inherited genetic variation can predict treatment-specific outcome following definitive prostate cancer treatment(43). Given the potential genetic heterogeneity in tumor tissue (44), the use of inherited variation to predict response is appealing, and could potentially allow for more tailored prostate cancer therapy. Indeed, our finding that variation in rs12757998 may impact the benefit of androgen deprivation therapy in men receiving radiation therapy is intriguing and worthy of further study. Additionally, androgen deprivation therapy has immune modulating properties (45) that could potentially synergize with the effects of radiotherapy, perhaps mitigating the effect of baseline immune status on disease outcome. 
There are some limitations to consider in interpreting our study findings. Although the majority of the recurrence data were confirmed from the medical records, those identified by self-report could potentially be less accurate despite the fact that our cohort consisted of well-educated health professionals. We did not perform any corrections for the testing of three individual SNPs. However, even after applying a conservative measure such as a Bonferroni correction, the association between variation in rs12757998 and biochemical recurrence would remain significant with a corrected alpha value of 0.02 and the association with the composite endpoint would be borderline. Regardless, validation of the association of variation in $R N A S E L$ on radiation treatment-specific prostate cancer outcomes in other populations is required. Additionally, since our analysis was limited to Caucasian patients, the generalizability to other races is uncertain. We did not assess complete coverage of potential variation across the $R N A S E L$ gene. Although we chose to sequence three $R N A S E L$ SNPs based on previous study findings $(16,19,35-37)$, including two known missense mutations, the mechanism by which rs 12757998 may act to increase circulating inflammatory mediators or impact radiation-response remains unknown, and this variant could potentially be a marker for the true causal variant. Also, although there were relatively few men who developed lethal prostate cancer, we cannot exclude the possibility that our inability to detect a statistically significant association between rs12757998 and lethal cancer was not the result of a differential biologic effect rather than a lack of power. Similarly, our secondary, exploratory analyses were likely underpowered to detect significant interaction-effects of treatment modality and brachytherapy on associations with rs12757998. However, we also cannot exclude the possibility that more events, particularly among men treated with brachytherapy, would have revealed a significant association between variation in rs12757998 and outcome in this group.

Despite these potential limitations, the current study represents one of the very few analyses performed to date examining the effect of inherited genetic variation on early-stage prostate cancer outcome that attempts to account for primary treatment (4). Given the prolonged natural history of early stage prostate cancer, the long-term and near complete follow-up for cancer outcomes including biochemical failure in our population was critical.

In summary, we have identified a polymorphism in the RNASEL gene that is statistically significantly associated with radiation treatment-specific outcome in HPFS participants diagnosed with early-stage prostate cancer. Although additional studies are needed to confirm this association and elucidate the functional significance of this polymorphism, our results suggest the immune system and inflammatory mediators could impact prostate cancer's response to radiation therapy. Future studies could potentially examine relationships between immune the systemic inflammatory response and radiation responsiveness in other malignancies. If confirmed, our results also suggest that genetic polymorphisms could eventually help better individualize treatment for early stage prostate cancer.

\section{Supplementary Material}

Refer to Web version on PubMed Central for supplementary material.

\section{Acknowledgments}

We thank the participants and staff of the Health Professionals Follow-up Study, for their valuable contributions as well as the following state cancer registries for their help: AL, AZ, AR, CA, CO, CT, DE, FL, GA, ID, IL, IN, IA, KY, LA, ME, MD, MA, MI, NE, NH, NJ, NY, NC, ND, OH, OK, OR, PA, RI, SC, TN, TX, VA, WA, WY.

FINANCIAL SUPPORT: JDS, JLK, and IMS were supported by NIH Training Grant 5 T32 CA 09001-36 and IMS was additionally supported by US Army Prostate Cancer Post-doctoral Fellowship; MME is supported by National 
Institutes of Health research training grant R25 CA098566; PLN was supported by a grant from an anonymous family foundation and LAM, PLN and NEM are supported by the Prostate Cancer Foundation. The project was supported by grants from the National Institutes of Health/National Cancer Institute (grant numbers R01 CA133891, R01CA141298-02, P01 CA055075)

\section{Works Cited}

1. National Comprehensive Cancer Network TNCC. NCCN Clinical Practice Guidelines in Oncology. 2009. NCCN Practice Guidelines in Oncology - Prostate Cancer; p. 1-46.v.2.2009

2. Sanda MG, Kaplan ID. A 64-year-old man with low-risk prostate cancer: review of prostate cancer treatment. JAMA. 2009; 301:2141-51. [PubMed: 19417179]

3. Barnett GC, West CM, Dunning AM, Elliott RM, Coles CE, Pharoah PD, et al. Normal tissue reactions to radiotherapy: towards tailoring treatment dose by genotype. Nature reviews Cancer. 2009; 9:134-42.

4. Borchiellini D, Etienne-Grimaldi M-C, Thariat J, Milano G. The impact of pharmacogenetics on radiation therapy outcome in cancer patients. A focus on DNA damage response genes. CANCER TREATMENT REVIEWS. 2012

5. Parliament MB, Murray D. Single nucleotide polymorphisms of DNA repair genes as predictors of radioresponse. Semin Radiat Oncol. 2010; 20:232-40. [PubMed: 20832015]

6. Burri RJ, Stock RG, Cesaretti JA, Atencio DP, Peters S, Peters CA, et al. Association of single nucleotide polymorphisms in SOD2, XRCC1 and XRCC3 with susceptibility for the development of adverse effects resulting from radiotherapy for prostate cancer. Radiation Research. 2008; 170:49-59. [PubMed: 18582155]

7. Cesaretti JA, Stock RG, Atencio DP, Peters SA, Peters CA, Burri RJ, et al. A genetically determined dose-volume histogram predicts for rectal bleeding among patients treated with prostate brachytherapy. Radiation Oncology Biology. 2007; 68:1410-6.

8. Cesaretti JA, Stock RG, Lehrer S, Atencio DA, Bernstein JL, Stone NN, et al. ATM sequence variants are predictive of adverse radiotherapy response among patients treated for prostate cancer. Radiation Oncology Biology. 2005; 61:196-202.

9. Kerns SL, Ostrer H, Stock R, Li W, Moore J, Pearlman A, et al. Genome-wide association study to identify single nucleotide polymorphisms (SNPs) associated with the development of erectile dysfunction in African-American men after radiotherapy for prostate cancer. Int J Radiat Oncol Biol Phys. 2010; 78:1292-300. [PubMed: 20932654]

10. Al-Ahmadi W, Al-Haj L, Al-Mohanna FA, Silverman RH, Khabar KSA. RNase L downmodulation of the RNA-binding protein, HuR, and cellular growth. Oncogene. 2009; 28:1782-91. [PubMed: 19252527]

11. Castelli J, Wood KA, Youle RJ. The 2-5A system in viral infection and apoptosis. Biomed Pharmacother. 1998; 52:386-90. [PubMed: 9856285]

12. Chakrabarti A, Jha BK, Silverman RH. New insights into the role of RNase L in innate immunity. Journal of Interferon \& Cytokine Research. 2011; 31:49-57. [PubMed: 21190483]

13. Perdiguero B, Esteban M. The interferon system and vaccinia virus evasion mechanisms. Journal of Interferon \& Cytokine Research. 2009; 29:581-98. [PubMed: 19708815]

14. Agalliu I, Leanza SM, Smith L, Trent JM, Carpten JD, Bailey-Wilson JE, et al. Contribution of HPC1 (RNASEL) and HPCX variants to prostate cancer in a founder population. Prostate. 2010; 70:1716-27. [PubMed: 20564318]

15. Carpten J, Nupponen N, Isaacs S, Sood R, Robbins C, Xu J, et al. Germline mutations in the ribonuclease L gene in families showing linkage with HPC1. Nat Genet. 2002; 30:181-4. [PubMed: 11799394]

16. Casey G, Neville PJ, Plummer SJ, Xiang Y, Krumroy LM, Klein EA, et al. RNASEL Arg462Gln variant is implicated in up to $13 \%$ of prostate cancer cases. Nat Genet. 2002; 32:581-3. [PubMed: 12415269]

17. Daugherty SE, Hayes RB, Yeager M, Andriole GL, Chatterjee N, Huang W-Y, et al. RNASEL Arg462Gln polymorphism and prostate cancer in PLCO. Prostate. 2007; 67:849-54. [PubMed: 17407163] 
18. Fesinmeyer MD, Kwon EM, Fu R, Ostrander EA, Stanford JL. Genetic variation in RNASEL and risk for prostate cancer in a population-based case-control study. Prostate. 2011

19. Meyer MS, Penney KL, Stark JR, Schumacher FR, Sesso HD, Loda M, et al. Genetic variation in RNASEL associated with prostate cancer risk and progression. Carcinogenesis. 2010; 31:1597603. [PubMed: 20576793]

20. Mi Y-Y, Zhu L-J, Wu S, Feng N-H. An update analysis of two polymorphisms in encoding ribonuclease $\mathrm{L}$ gene and prostate cancer risk: involving 13,372 cases and 11,953 controls. Genes Nutr. 2011; 6:397-402. [PubMed: 21499914]

21. Rennert H, Bercovich D, Hubert A, Abeliovich D, Rozovsky U, Bar-Shira A, et al. A novel founder mutation in the RNASEL gene, 471delAAAG, is associated with prostate cancer in Ashkenazi Jews. Am J Hum Genet. 2002; 71:981-4. [PubMed: 12145743]

22. Robbins CM, Hernandez W, Ahaghotu C, Bennett J, Hoke G, Mason T, et al. Association of HPC2/ELAC2 and RNASEL non-synonymous variants with prostate cancer risk in African American familial and sporadic cases. Prostate. 2008; 68:1790-7. [PubMed: 18767027]

23. Rökman A, Ikonen T, Seppälä EH, Nupponen N, Autio V, Mononen N, et al. Germline alterations of the RNASEL gene, a candidate HPC1 gene at 1q25, in patients and families with prostate cancer. Am J Hum Genet. 2002; 70:1299-304. [PubMed: 11941539]

24. Shook SJ, Beuten J, Torkko KC, Johnson-Pais TL, Troyer DA, Thompson IM, et al. Association of RNASEL variants with prostate cancer risk in Hispanic Caucasians and African Americans. Clin Cancer Res. 2007; 13:5959-64. [PubMed: 17908993]

25. Wang L, Mcdonnell SK, Elkins DA, Slager SL, Christensen E, Marks AF, et al. Analysis of the RNASEL gene in familial and sporadic prostate cancer. Am J Hum Genet. 2002; 71:116-23. [PubMed: 12022038]

26. Wiklund F, Jonsson B-A, Brookes AJ, Strömqvist L, Adolfsson J, Emanuelsson M, et al. Genetic analysis of the RNASEL gene in hereditary, familial, and sporadic prostate cancer. Clin Cancer Res. 2004; 10:7150-6. [PubMed: 15534086]

27. Hatfield P, Merrick A, Harrington K, Vile R, Bateman A, Selby P, et al. Radiation-induced cell death and dendritic cells: potential for cancer immunotherapy? Clinical Oncology. 2005; 17:1-11. [PubMed: 15714922]

28. Levy A, Chargari C, Cheminant M, Simon N, Bourgier C, Deutsch E. Radiation therapy and immunotherapy: Implications for a combined cancer treatment. Critical reviews in oncology/ hematology. 2012

29. Lee Y, Auh SL, Wang Y, Burnette B, Wang Y, Meng Y, et al. Therapeutic effects of ablative radiation on local tumor require $\mathrm{CD} 8+\mathrm{T}$ cells: changing strategies for cancer treatment. Blood. 2009; 114:589-95. [PubMed: 19349616]

30. Stone HB, Peters LJ, Milas L. Effect of host immune capability on radiocurability and subsequent transplantability of a murine fibrosarcoma. Journal of the National Cancer Institute. 1979; 63:1229-35. [PubMed: 291749]

31. Christensen E, Pintilie M, Evans KR, Lenarduzzi M, Ménard C, Catton CN, et al. Longitudinal cytokine expression during IMRT for prostate cancer and acute treatment toxicity. Clin Cancer Res. 2009; 15:5576-83. [PubMed: 19706801]

32. Hurwitz MD, Kaur P, Nagaraja GM, Bausero MA, Manola J, Asea A. Radiation therapy induces circulating serum Hsp72 in patients with prostate cancer. Radiother Oncol. 2010; 95:350-8. [PubMed: 20430459]

33. Johnke RM, Edwards JM, Evans MJ, Nangami GN, Bakken NTG, Kilburn JM, et al. Circulating cytokine levels in prostate cancer patients undergoing radiation therapy: influence of neoadjuvant total androgen suppression. In Vivo. 2009; 23:827-33. [PubMed: 19779119]

34. Lopes CO, Callera F. Three-Dimensional Conformal Radiotherapy in Prostate Cancer Patients: Rise in Interleukin 6 (IL-6) but not IL-2, IL-4, IL-5, Tumor Necrosis Factor-a, MIP-1-a, and LIF Levels. International journal of radiation oncology, biology, physics. 2011

35. Breyer JP, McReynolds KM, Yaspan BL, Bradley KM, Dupont WD, Smith JR. Genetic variants and prostate cancer risk: candidate replication and exploration of viral restriction genes. Cancer epidemiology, biomarkers \& prevention: a publication of the American Association for Cancer Research, cosponsored by the American Society of Preventive Oncology. 2009; 18:2137-44. 
36. Li H, Tai BC. RNASEL gene polymorphisms and the risk of prostate cancer: a meta-analysis. Clin Cancer Res. 2006; 12:5713-9. [PubMed: 17020975]

37. Noonan-Wheeler FC, Wu W, Roehl KA, Klim A, Haugen J, Suarez BK, et al. Association of hereditary prostate cancer gene polymorphic variants with sporadic aggressive prostate carcinoma. Prostate. 2006; 66:49-56. [PubMed: 16114055]

38. Kenfield SA, Stampfer MJ, Chan JM, Giovannucci E. Smoking and prostate cancer survival and recurrence. JAMA. 2011; 305:2548-55. [PubMed: 21693743]

39. Malathi K, Paranjape JM, Ganapathi R, Silverman RH. HPC1/RNASEL mediates apoptosis of prostate cancer cells treated with $2^{\prime}, 5^{\prime}$-oligoadenylates, topoisomerase I inhibitors, and tumor necrosis factor-related apoptosis-inducing ligand. Cancer Res. 2004; 64:9144-51. [PubMed: 15604285]

40. Weichselbaum RR, Ishwaran H, Yoon T, Nuyten DS, Baker SW, Khodarev N, et al. An interferonrelated gene signature for DNA damage resistance is a predictive marker for chemotherapy and radiation for breast cancer. Proceedings of the National Academy of Sciences of the United States of America. 2008; 105:18490-5. [PubMed: 19001271]

41. Burnette BC, Liang H, Lee Y, Chlewicki L, Khodarev NN, Weichselbaum RR, et al. The efficacy of radiotherapy relies upon induction of type i interferon-dependent innate and adaptive immunity. Cancer Res. 2011; 71:2488-96. [PubMed: 21300764]

42. Coate L, Cuffe S, Horgan A, Hung RJ, Christiani D, Liu G. Germline genetic variation, cancer outcome, and pharmacogenetics. J Clin Oncol. 2010; 28:4029-37. [PubMed: 20679599]

43. Gao R, Price DK, Dahut WL, Reed E, Figg WD. Genetic polymorphisms in XRCC1 associated with radiation therapy in prostate cancer. Cancer Biol Ther. 2010; 10:13-8. [PubMed: 20495366]

44. Gerlinger M, Rowan AJ, Horswell S, Larkin J, Endesfelder D, Gronroos E, et al. Intratumor heterogeneity and branched evolution revealed by multiregion sequencing. N Engl J Med. 2012; 366:883-92. [PubMed: 22397650]

45. Aragon-Ching JB, Williams KM, Gulley JL. Impact of androgen-deprivation therapy on the immune system: implications for combination therapy of prostate cancer. Frontiers in bioscience: a journal and virtual library. 2007; 12:4957-71. [PubMed: 17569623] 


\section{Translational Relevance}

Radiation therapy is an accepted treatment for localized prostate cancer; however, there are no commonly used factors that predict outcome specifically following this treatment. In this article, we observed an association between variation in a biologically relevant single nucleotide polymorphism in the RNASEL gene and outcome following prostate radiotherapy in a large cohort of men enrolled in the prospective US Health Professionals Follow-Up Study that were extensively followed for cancer-specific outcomes. This finding remained significant after adjusting for other known prognostic factors, and was limited to patients who received external beam radiation as opposed to brachytherapy. Moreover, this association was not found in patients derived from the same cohort treated with radical prostatectomy instead of radiotherapy. These data are of translational relevance because they generate the hypothesis that inherited variation in RNASEL may predict outcome following radiotherapy in a treatment-specific manner that could eventually be used to help guide treatment. 


\section{Table 1}

Characteristics among men with prostate cancer $(n=434)$ in the Health Professionals Follow-up Study who underwent radiation therapy

\begin{tabular}{|c|c|}
\hline Median Age at diagnosis (IQR) & 72 yrs $(68-76$ yrs $)$ \\
\hline Median Age at XRT (IQR) & 72 yrs $(69-77 \mathrm{yrs})$ \\
\hline \multicolumn{2}{|l|}{ PSA at diagnosis, $\mathbf{N}(\%)$} \\
\hline$<10$ & $312(73)$ \\
\hline $10-20$ & $95(22)$ \\
\hline $20-50$ & $22(5)$ \\
\hline Unknown & 5 \\
\hline \multicolumn{2}{|l|}{ Gleason score, N (\%) } \\
\hline$<7$ & $292(69)$ \\
\hline 7 & $95(22)$ \\
\hline$>7$ & $36(9)$ \\
\hline Unknown & 11 \\
\hline \multicolumn{2}{|l|}{ Clinical Stage, N (\%) } \\
\hline $\mathrm{T} 1 / \mathrm{T} 2$ & $418(98)$ \\
\hline $\mathrm{T} 3$ & $10(2)$ \\
\hline Unknown & 6 \\
\hline \multicolumn{2}{|l|}{ Radiation Treatment, $\mathbf{N}$} \\
\hline External beam & $243(56)$ \\
\hline Brachytherapy & $143(33)$ \\
\hline External beam + Brachytherapy & $48(11)$ \\
\hline Adjuvant/neoadjuvant ADT, N (\%) & $173(40 \%)$ \\
\hline Reached composite outcome, N (\%) & $100(23 \%)$ \\
\hline Biochemical recurrence only & $77(77 \%)$ \\
\hline Lethal prostate cancer only & $9(9 \%)$ \\
\hline Biochemical recurrence/lethal prostate cancer & $14(14 \%)$ \\
\hline \multicolumn{2}{|l|}{$\underline{\text { Variation in }} \underline{R N A S E L}$} \\
\hline \multicolumn{2}{|l|}{ rs486907 (R462Q), N (\%) } \\
\hline $\mathrm{CC}$ & $177(41)$ \\
\hline $\mathrm{CT}$ & $195(45)$ \\
\hline TT & $57(13)$ \\
\hline Unknown & 5 \\
\hline \multicolumn{2}{|l|}{ rs627928 (D541E), N (\%) } \\
\hline AA & $134(32)$ \\
\hline $\mathrm{AC}$ & $208(49)$ \\
\hline $\mathrm{CC}$ & $82(19)$ \\
\hline
\end{tabular}




\begin{tabular}{|l|c|} 
Unknown & 10 \\
\hline rs12757998 (\%), N (\%) & \\
GG & $218(51)$ \\
GA & $176(42)$ \\
AA & $30(7)$ \\
Unknown & 10 \\
\hline
\end{tabular}

Abbreviations: yrs - years; IQR - interquartile range; XRT - radiation therapy; PSA - prostate-specific antigen; ADT - androgen deprivation therapy; R - arginine; Q - glutamine; E - glutamic acid; D - aspartic acid; C - cytosine; T - thymine; A - adenine 
Table 2

Multivariate hazard ratios of associations between SNPs in RNASEL and outcomes after radiation therapy, Health Professionals Follow-up Study *

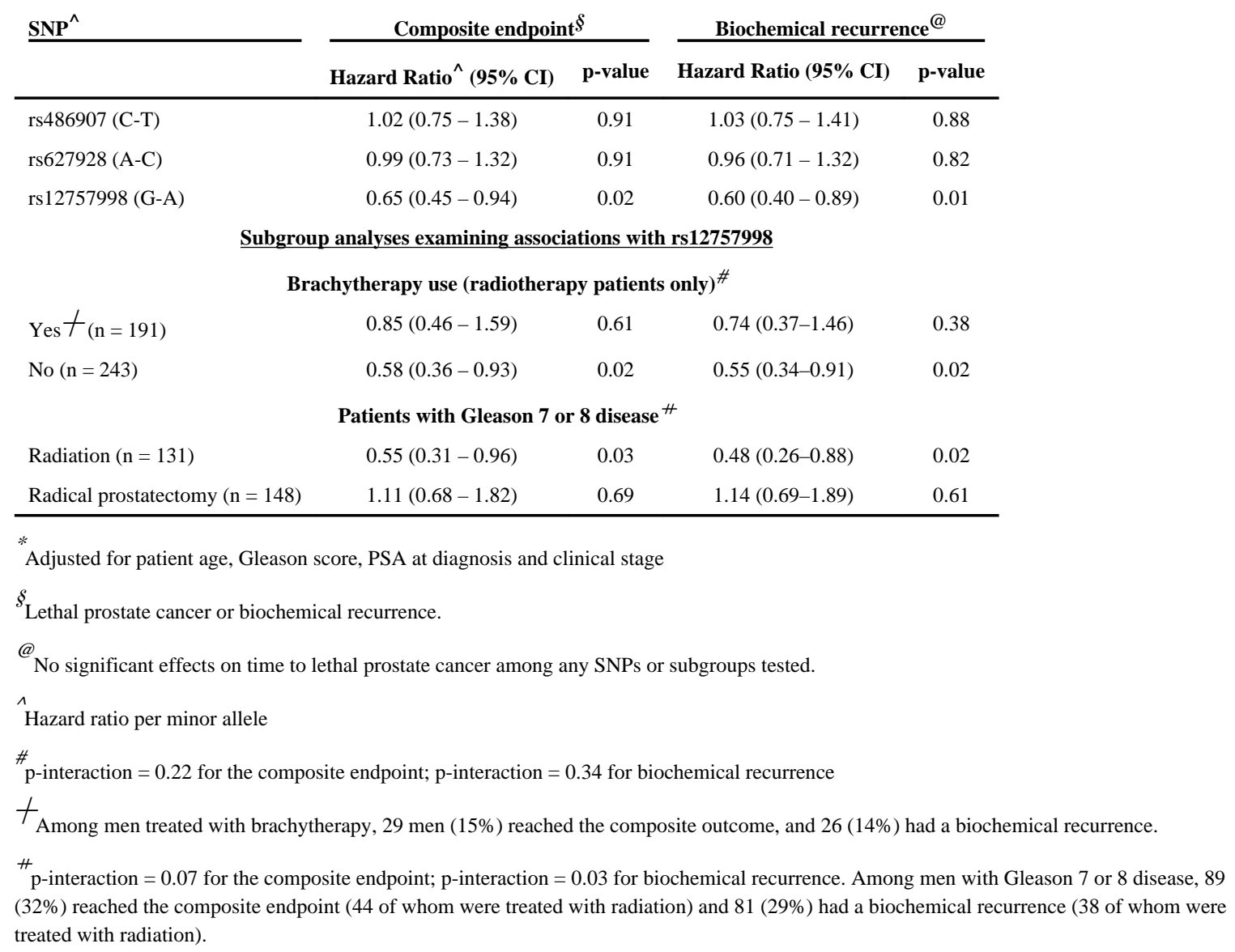

\begin{tabular}{lccccc} 
& Hazard Ratio $^{\wedge}(\mathbf{9 5 \%} \mathbf{C I})$ & p-value & & Hazard Ratio (95\% CI) & p-value \\
\hline rs486907 (C-T) & $1.02(0.75-1.38)$ & 0.91 & $1.03(0.75-1.41)$ & 0.88 \\
rs627928 (A-C) & $0.99(0.73-1.32)$ & 0.91 & $0.96(0.71-1.32)$ & 0.82 \\
rs12757998 (G-A) & $0.65(0.45-0.94)$ & 0.02 & $0.60(0.40-0.89)$ & 0.01
\end{tabular}

Subgroup analyses examining associations with rs12757998

Brachytherapy use (radiotherapy patients only) ${ }^{\#}$

$\begin{array}{lllll}\text { Yes } f_{(\mathrm{n}=191)} & 0.85(0.46-1.59) & 0.61 & 0.74(0.37-1.46) & 0.38 \\ \text { No }(\mathrm{n}=243) & 0.58(0.36-0.93) & 0.02 & 0.55(0.34-0.91) & 0.02\end{array}$

No $(\mathrm{n}=243)$

Patients with Gleason 7 or 8 disease ${ }^{*}$

Radiation $(\mathrm{n}=131)$

$\begin{array}{llll}0.55(0.31-0.96) & 0.03 & 0.48(0.26-0.88) & 0.02\end{array}$

Radical prostatectomy $(\mathrm{n}=148)$

$1.11(0.68-1.82)$

0.69

$1.14(0.69-1.89)$

0.61

* Adjusted for patient age, Gleason score, PSA at diagnosis and clinical stage

$\xi_{\text {Lethal prostate cancer or biochemical recurrence. }}$

${ }^{\circledR}$ No significant effects on time to lethal prostate cancer among any SNPs or subgroups tested.

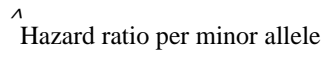

${ }^{\#}$-interaction $=0.22$ for the composite endpoint; $\mathrm{p}$-interaction $=0.34$ for biochemical recurrence

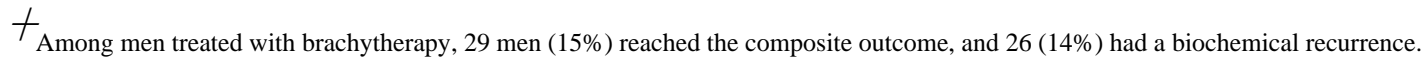

${ }^{*}$ p-interaction $=0.07$ for the composite endpoint; p-interaction $=0.03$ for biochemical recurrence. Among men with Gleason 7 or 8 disease, 89 (32\%) reached the composite endpoint (44 of whom were treated with radiation) and $81(29 \%)$ had a biochemical recurrence (38 of whom were treated with radiation).

Abbreviations: SNP - single nucleotide polymorphism; PSA - prostate-specific antigen; CI - confidence interval 
Table 3

Association between variation in rs12757998 and outcomes following androgen deprivation therapy*

\begin{tabular}{|c|c|c|c|c|}
\hline \multirow[t]{2}{*}{ Effects of ADT } & \multicolumn{2}{|c|}{ Composite endpoint $\S$} & \multicolumn{2}{|c|}{ Biochemical recurrence@ } \\
\hline & Hazard Ratio (95\% CI) & p-value & Hazard Ratio (95\% CI) & p-value \\
\hline In men with rs 12757998 major allele (G) & $0.38(0.20-0.72)$ & 0.003 & $0.42(0.22-0.79)$ & 0.007 \\
\hline In men with rs 12757998 variant allele $(\mathrm{A})^{\wedge}$ & $1.34(0.69-2.59)$ & 0.39 & $1.64(0.80-3.36)$ & 0.18 \\
\hline \multicolumn{5}{|c|}{ Adjusted for patient age, Gleason score, PSA at diagnosis and clinical stage } \\
\hline \multicolumn{5}{|c|}{${ }^{\#}$ p-interaction $=0.02$ for the composite endpoint; $\mathrm{p}$-interaction $=0.01$ for biochemical recurrence (variant allele considered additively) } \\
\hline \multicolumn{5}{|l|}{${ }^{\S}$ Lethal prostate cancer or biochemical recurrence } \\
\hline \multicolumn{5}{|l|}{ At least one variant allele } \\
\hline \multicolumn{5}{|c|}{${ }^{@}$ No significant effects on time to lethal prostate cancer. } \\
\hline
\end{tabular}

